\title{
Prediction of diabetic retinopathy using machine learning techniques
}

DOI:10.36909/jer.13947

T Jemima Jebaseeli*, C. Anand Deva Durai**, Salem Alelyani***, Mohammed Saleh Alsaqer $^{* * * *}$

*Assistant Professor, Department of Computer Science and Engineering, Karunya Institute of Technology and Sciences, Coimbatore, Tamilnadu, India.

**Assistant Professor, College of Computer Science, King Khalid University, Abha, Kingdom of Saudi Arabia.

**** Director of Research and Development Center for Artificial Intelligence, College of Computer Science, King Khalid University, Abha, Kingdom of Saudi Arabia.

${ }^{* * * *}$ Head of Research and Development Center for Artificial Intelligence, College of Computer Science, King Khalid University, Abha, Kingdom of Saudi Arabia.

*Email: jemima_jeba@karunya.edu; Corresponding Author.

\begin{abstract}
Diabetic Retinopathy (DR) is the complicatedness of diabetes that happens due to macular degeneration among Type II diabetic patients. The early symptom of this disease is predicted through annual eye checkups. Hence, one can save their vision at an early stage. Later on, it prompts retinal detachment. There is a requirement for awareness among diabetic patients about this disease to prevent their life from vision misfortune. Along these lines, there is a need for a computer-assisted method to analyze the disease. The proposed system used Adaptive Histogram Equalization (AHE) technique for image enhancement, Hop Field Neural Network
\end{abstract}


for blood vessel segmentation, and Adaptive Resonance Theory (ART) for blood vessel classification. The proposed system analyzes the disease and classifies the disease level effectively with high accuracy. Also, the system notifies the users about the stages of the disease. The proposed system is evaluated with the clinical as well as open fundus image data sets like DRIVE, STARE, MESSIDOR, HRF, DRIONS, and REVIEW for diabetic retinopathy prediction. Also, physicians evaluated the system and concluded that the proposed system does not deviate from the quality of disease analysis and grading. The proposed techniques accomplished $99.99 \%$ accuracy. The system is evaluated by the ophthalmologists and witnesses that the proposed system has not veered off as far as quality.

Keywords: diabetic retinopathy; fundus image; diabetes; segmentation; classification; feature extraction.

\section{INTRODUCTION}

Diabetes occurs due to an increase in the glucose levels in the human body. While insulin is not segregated enough according to the human body, then it is known as Type I diabetes. If the expected insulin is segregated more than the level then it is known as Type II Diabetes. Diabetic retinopathy occurs among patients with Type II diabetes (Varun et al., 2016). It affects the retina in human beings. The retina is a vital organ in humans for vision. The light signal passes through the retina to the brain. Diabetic retinopathy affects the macula and causes degeneration. The blood vessels inside the retina are called arteries and veins which is the reason for blood flow. Due to diabetic retinopathy, the blood vessels cannot carry blood into the retina. When the patients rubbed their eyes, the blood vessels started to burst and settle the proteins and lipids inside the retina as sores such as microaneurysm, cotton wool spots, and exudates. It causes virtuous hemorrhages in the retina (Francis et al., 2016). At the same time, the new blood vessels growth that happens inside the retina is called neovascularization that strives to carry blood flow makes the retina worse. Despite retinal vessel impediments, new 
vessels forming into the retina ought to be broken down. Hence, the patient's vision got blurred, and could not recognize objects. At the early stage, this can be treated through photocoagulation and laser treatments. Later, it makes the patients $100 \%$ blind. In diabetic retinopathy, there are two classifications: NPDR and PDR (Jemima et al., 2018). Nonproliferative DR is the primary period and proliferative is the advanced stage. Depends on the number of microaneurysms, hard and soft exudates, red and white lesions, the disease is graded as mild level, moderate level, or severe level. Analyzing the image by the human vision is critical and consumes more. While analyzing fundus images of the retina, there are intensitywise variations between the tiny blood vessel and background image. The retinal depigmentation images are delegated and preprocessing may meld the vessels and it is hard to portion them out from the background. If there is an error in identifying the original blood vessel may lead to the formation of the wrong vessel vasculature. Based on this inspiration, the proposed method is capable in segment the blood vessel at their cross limits if it is smothered with some other variations from the norm. A detailed survey on various issues in the retinal blood vessel and lesion segmentation has been made and the findings of the investigation are given as relative examination. The various retinal blood vessel and lesion segmentation methodologies proposed as of now are well suited for any sort of fundus image database. Most of the segmentation algorithms are reliant upon the accuracy of the preprocessed image inputs (Gwenole et al., 2008). Various researchers proposed algorithms for the extraction of blood vessels from the retina. Regardless, these procedures work just on fundus pictures with no fanatical effects. George Azzopardi et al., 2015 plotted a BCOSFIRE channel. The execution of the channel is impacted by its boundaries. Wilfred Franklin et al., 2014 used the multilayer perceptron model. The model achieved less precision value due to its pixel processing approach. Geetha Ramani et al., 2016 used PCA and k-means techniques. The algorithm produced the best precision value, but sensitivity is greatly reduced. Rajaby et al., 2016 
presented an NWFCM calculation to find the blood vessels and track the vessel direction. The nonlinear technique causes issues in the transmuted space from the low dimensional space in tracking the vessels due to its complex topology.

The following challenges are faced while segmenting the exudates in the literature. Carla et al., 2015 have identified an ant colony optimization technique for exudate segmentation. The algorithm couldn't detect the exudates which are not matched by the target threshold value. Also, as needed by clinical practices, the algorithm needs improvements. The algorithm is rigid and cannot be generalized for unknown situations. All the surveyed papers are trying to obtain precise segmentation results. The significance and the adaptive nature of the proposed Hop Field Neural Network and ART1 have to enhance the segmentation results than other methods. The Adaptive Resonance Theory improves the classification of DR in an unsupervised manner for the early prediction of DR. The proposed method is not confused with the features of hemorrhages and microaneurysms. The network is successfully locating the large pathological components as well.

\section{METHOD}

The fundus images are used for the diagnosis of DR. The publically available fundus images are captured by technicians at different Fields of View (FoV). It is the optical angle of view of the lens in fundus cameras. An angle of $30^{\circ}$ is 2.5 times the size of a film's picture as compared to the typical viewing angle. Wide-angle fundus cameras capture images between $45^{\circ}$ and $140^{\circ}$ and give the retinal enlargement proportionally lower. An angle of vision of $20^{\circ}$ or less in a narrow fundus camera. But these fundus images are captured at different illumination and contain noise. This can be eliminated through Image enhancement techniques 
Table 1 Fundus image dataset for experiments.

\begin{tabular}{cccc}
\hline Database & Dimension (pixels) & No. of Images & Infected Images \\
\hline DRIVE & $565 \times 584$ & 40 & 7 \\
STARE & $700 \times 605$ & 400 & 338 \\
REVIEW & $2160 \times 1440$ & 16 & 7 \\
HRF & $3504 \times 2336$ & 15 & - \\
DRIONS & $600 \times 400$ & 110 & 8
\end{tabular}

For research purposes, there are publically available datasets: DRIVE, STARE, MESSIDOR, HRF, DRIONS, and REVIEW. The dimensions of these datasets are given in Table 1. There are 581 pictures utilized for DR experimental analysis.

\section{PROPOSED ARCHITECTURE OF THE BLOOD VESSEL SEGMENTATION}

The Hop Field Neural Network algorithm measures the width of retinal vessels of the fundus photographs and segments the vessel edges simultaneously. As depicted in Figure 1, the proposed architecture has five primary phases: (1) Mask is generated automatically and applied over the image. The masking process removes the background pixels, (2) Quality of the image is assessed, to reject the poor quality images, (3) Segmentation of blood vessels. (4) Identify and track the vascular map, (4) Classification of blood vessels from non-blood vessels, (5) Grading of DR. The strategy infers the utilization of different image processing techniques such as histogram matching, edges detection, Hop Field Neural Network, and ART, etc. 


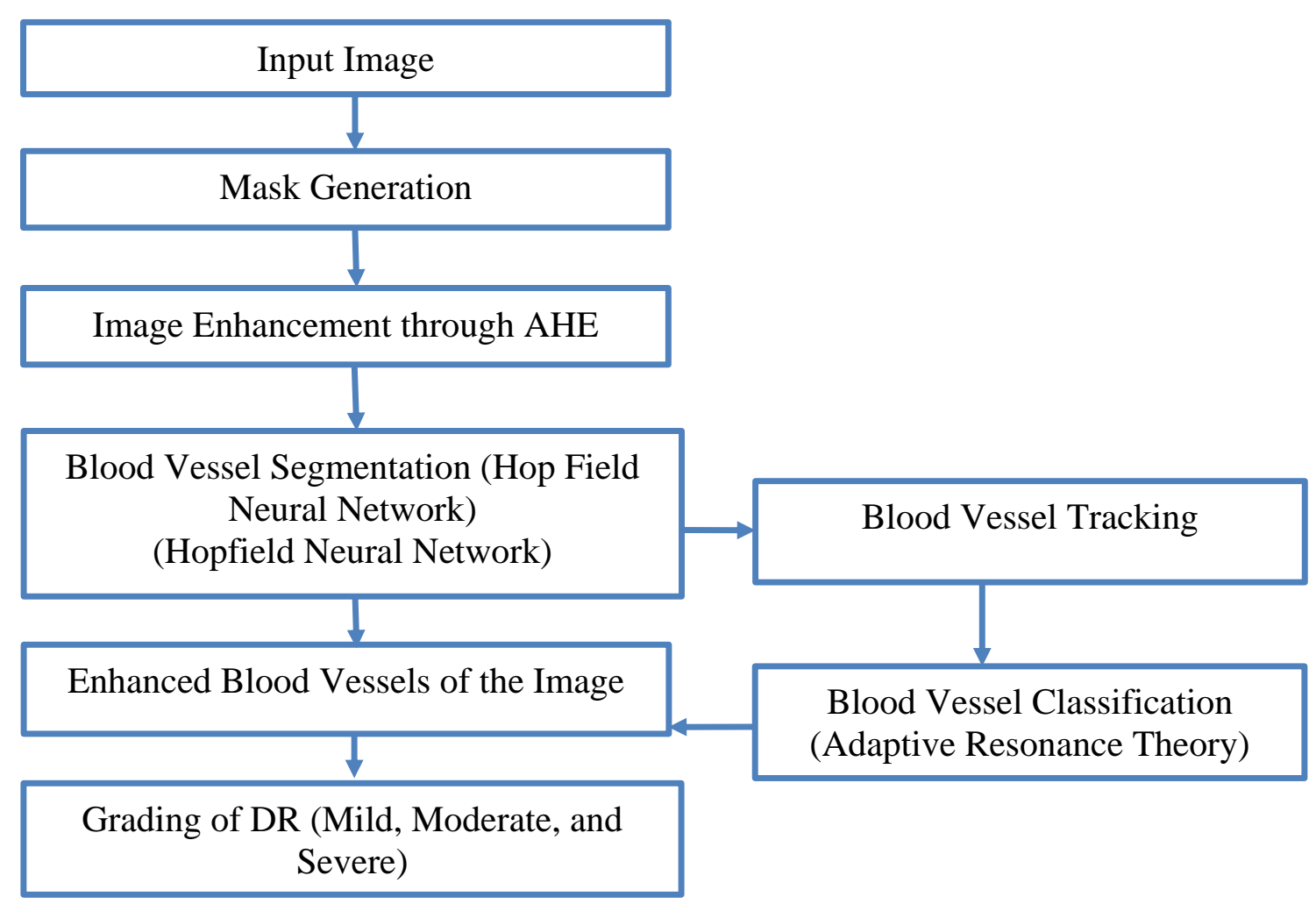

Figure 1 The proposed architecture to diagnose DR.

\section{FUNDUS IMAGE PREPROCESSING}

The captured images are affected by the illuminations of the camera. The fundus camera will be tilted to the different FoV to capture the right and left eyes of the patients. The proposed system uses the Adaptive Histogram Equalization (AHE) technique for enhancement. AHE is a common approach for optimizing fundus picture gray scale intensity. The probability distribution function dynamically expands the gray picture to increase the intensity of the blood vessel pixels' green channel. The global histogram technique of equalization enhances the preprocessing speed. The method automatically determines the gray picture level and adjusts the optimum histogram value between neighboring gray level pixels as per the threshold. The histogram equalization technique uses gray pixels and transforms the histogram to a single one with distinct gray levels and continuous smoothness so that a picture may be created. Consider the gray level value of the input fundus image is $I_{G}$ and the probability density 
function of the image is $P\left(I_{G}\right)$. Then $P\left(I_{E}\right) d_{I E}=P\left(I_{G}\right) d_{I G}$ is the final contrastenhanced histogram image.

\section{MASK GENERATION}

The mask generation is the procedure where the pixels that have a place within ROI are marked as vessel pixels. The pixels outside that ROI encompass the region with dark dull with 0 intensity value and it is required to be disposed of before passing the images through the next phase.

\section{HOP FIELD NEURAL NETWORK}

Hopfield Neural Networks are developed from the artificial neural network neurons. These neurons have $\mathrm{N}$ inputs. As shown in Figure 2, for every input $i, w_{i}$ is the associated weight. The estimation of each input $x_{i}$ is determined and the aggregated weight is equal then $w_{i} x_{i}$ is determined.

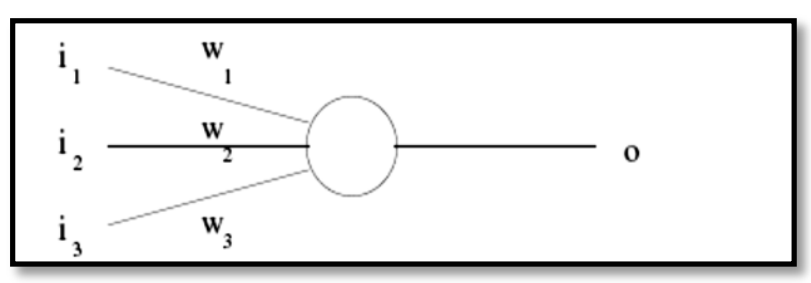

Figure 2 Structure of Hopfield Neural network.

A Hopfield network is a neural network system framework that comprises of $N$ neurons. The corresponding weight is applied to the system from $j$ to $i$ neuron is considered as $w_{i j}$. This weight is represented as a matrix $w$. The component of weight matrix is represented as $w_{i j}$. So, the weight matrix $w$, refreshing principle of neurons, and the neuron's movement in the system are based on the characterized rules. There are two different ways of refreshing the network. In the asynchronous method where the network picks one neuron, then computes the total input weight and updates it right away. In the synchronous method, the aggregate of input weighted of the corresponding neuron is updated without the refreshment of other neurons. The pattern is 
programmed by assigning the specific value to all nodes or the specific node. The value of the node is updated once for all iteration by the asynchronous or synchronous mode. The emphases are halted when it arrives at the greatest threshold. The neuron looks for corresponding patterns to produce the output. Hop Field Neural Network generates the pattern and represents it by a weight matrix. These patterns are represented in two ways: symmetry and no self-associations. The neurons find the symmetry patterns then its weight matrix is updated as $w_{i j}=w_{j i}$. If the neurons find the no self-association mode then its weight will be set it as $w_{i i}=0$

\section{WEIGHT MATRIX GENERATION FOR A PATTERN}

There are two neurons in the network, in that if the weight of two neurons is positive then, both neurons move together in the same direction. It is likewise evident in bigger networks. Assume that the network have neuron $i$ associated with neuron $j$ with a weight of +1 , at that point the commitment of neuron $i$ to the weighted input entirety of neuron $j$ is certain if $x_{i}=1$ and negative if $x=-1$. It is represented by,

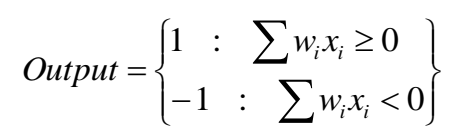

At the end of the iteration, neuron $i$ attempt to drive neuron $j$ to a similar incentive as it has at present. On the off chance that the association loads between them are negative, in any case, neuron $i$ will attempt to drive neuron $j$ to the contrary worth. The network has the option to store the uncorrupted design as it has a memory. In such a case, the systems are called Hopfield memories.

\section{ADAPTIVE RESONANCE THEORY - 1 FRAMEWORK}

The ART1 framework comprises two significant subsystems, an attentional subsystem, and an orienting subsystem. The framework designs the coordinating activity during which the system structure attempts to decide if the input design is among the patterns that are recently put away in the network or not. The attentional subsystem has the following features: 
- The input neurons of the F1 layer are the correlation layer of transient memory.

- The output neurons of the F2 layer are an acknowledgment layer of short-term memory.

- The gain control unit, Gain 1 and Gain 2, one for each layer.

The subsystem has the Long Term Memory (LTM) with the following features:

- Interconnections among the nodes in each layer have not appeared.

- Inhibitory association of -ve weights for gain control from the F2 layer.

- To constitute excitation association of +ve weights from gain control to F1 and F2.

The subsystem has the Situating subsystem with the following features:

The situating subsystem contains the accompanying layers:

- $\quad$ Reset layer for controlling the attentional subsystem by and large elements.

- Inhibitory association of -ve weights from the F1 layer to reset node.

- Excitatory association of +ve weights from the reset node to the F2 layer.

\section{ART1 PATTERN MATCHING CYCLE}

The ART network structure designs coordinating and attempts to decide if an input design is among the patterns that are recently put away in the system or not. Pattern matching technique comprises input pattern, pattern matching techniques, reset activities, and final acknowledgment. As per Figure 3, the principle design $\mathrm{x}$ is restored on F1 and another pattern of coordination starts. In this manner, another pattern $Y$ is restored on F2. The generation of patterns continues till it find the perfect match for the pattern input, or this process stops till the F2 layer produces the patterns. 


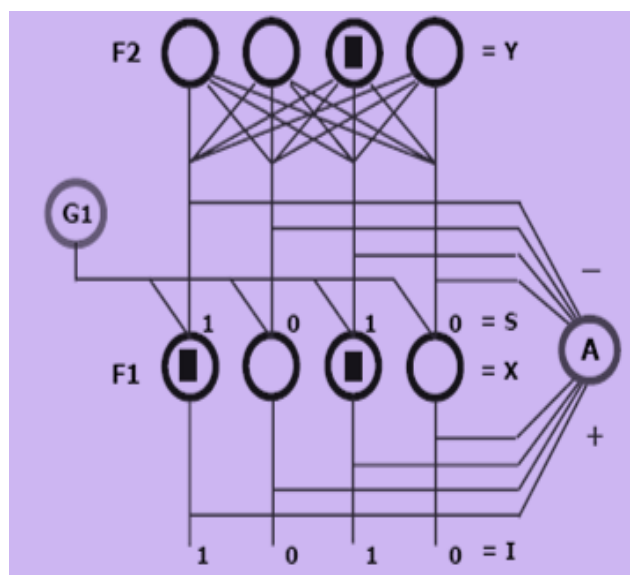

(a)

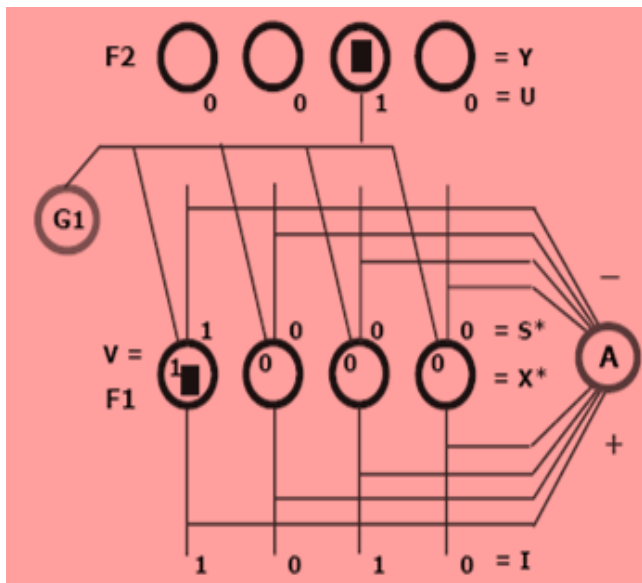

(b)

Figure 3 ART1 system: (a) Input pattern, (b) Pattern matching.

In case no match is found, the framework will generate the new pattern by disposing of the uncertain F2 layer's nodes. The learning process happening at LTM updates the weights. These training processes continuously process the input and generate the pattern. If any signals pass over the association state, then the weights of the associations are modified by the generated pattern. The jumbles don't bring about loss of information or learning of the wrong affiliation in light. The time required to generate the weights are depends on the overall coordination of inputs and patterns in the associated network. The association taking an interest in a mismatch is not enough to impact the related weights genuinely. At the point when the match happens, the reset signal will not be generated to reset the state. Also, the generated associated patterns for the corresponding inputs are dynamic and that stay a long time in the network till the weight is finalized by the network. This process continues for the F2 layer to get the required matched pattern. The jumbles don't realize the loss of data or learning of an inappropriate connection concerning organized cycle time and time required to change the weights. The association looking interested in a mismatch is not enough to impact the related weights genuinely. Right when the match occurs, the iterations don't generate the reset signal. The associations never stop until it generates the perfect pattern. This could be done by delaying the process of dynamic pattern generation by strengthening the weights. 


\section{RESULTS AND ANALYSIS}

The key inspiration of the proposed strategy is to improve the nature of fundus pictures by enhancement and segmentation. Despite huge research in this domain, there is a need for precise prediction of malignant for clinical diagnosis. Grounded on this inspiration, the programmed retinal vein segmentation and lesions strategies are introduced in the proposed work. The proposed technique considered around 581 images of different public datasets. Figure 4 shows the segmentation of lesions.

Then the infected retinal images are graded after segmentation as follows:

Case 1: If there is only one microaneurysm present in the image then the grade is very mild.

Case 2: If there are microaneurysms, retinal hemorrhages, exudates, and cotton wool spots then it is grade as mild.

Case 3: In the moderate case, where venous beading will be in multiple quadrants, hemorrhages in one to three quadrants, along with the presence of cotton wool spots.

Case 4: If there are huge amounts of hemorrhages in all quadrants, venous beading in not less than two quadrants, then it is graded as severe.

Case 5: At a very severe stage, lesions will be in more than two quadrants.

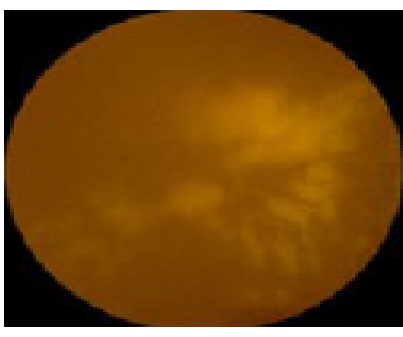

(a)

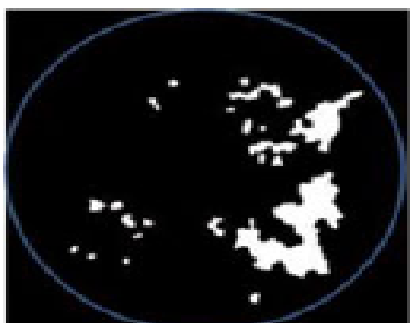

(b)

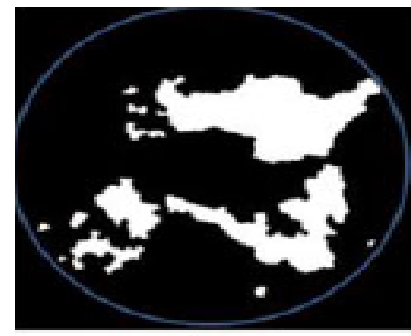

(c)

Figure 4 (a) Image with depigmentation (b) \& (c) Segmentation of lesions.
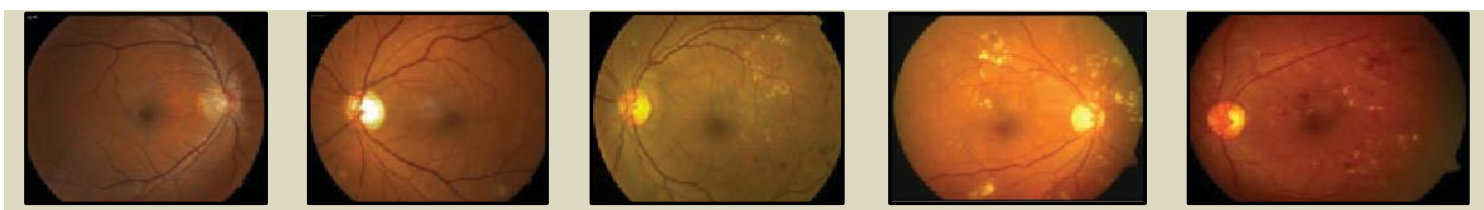


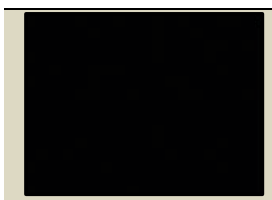

(a)

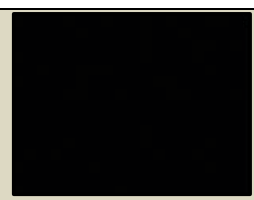

(b)

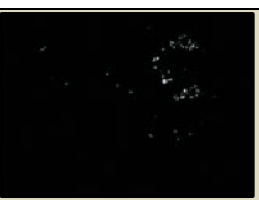

(c)

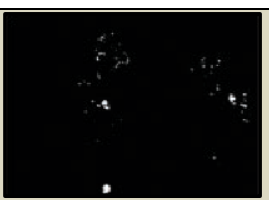

(d)

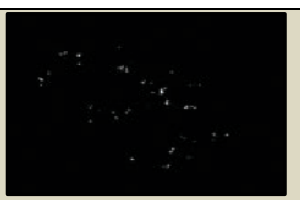

(e)

Figure 5 Grading of diabetic retinopathy: (a) Standard Retina (b) Mild case, (c) Moderate case,

(d) Severe case, (e) Very severe case.

As shown in Figure 5, in the mild stage, microaneurysms, hemorrhages, exudates, lesions are presented in any or all parts of the image. At the moderate stage, in the first three quadrants there will be retinal hemorrhages, and in more than one quadrant there will be venous beading along with cotton wool spots. The severe stage contains severe retinal hemorrhages in all quadrants, significant venous beading in more than two quadrants.

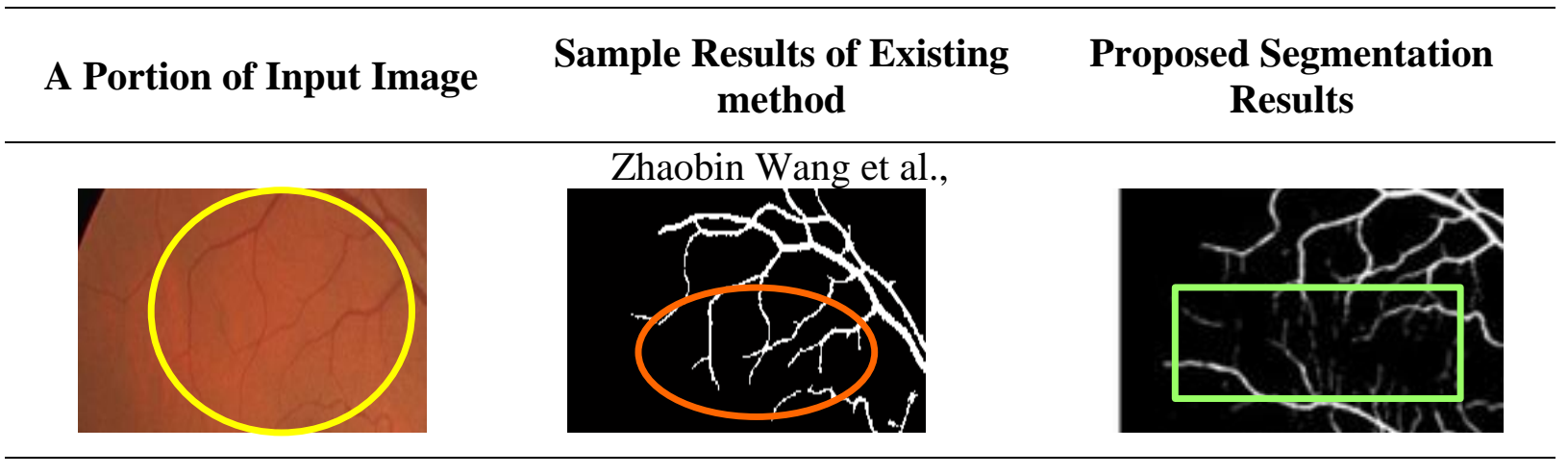

Figure 6. Comparison of tiny vessel detection with competitive methods.

As per Figure 6, column-1 indicates the source image with small vessels that are hard to detect, column-2 shows the methodology failed in identifying small vessels by the competitive methods, and column-3 shows the small vessels hard to detect are discovered by the proposed method.

\section{PERFORMANCE METRICS}

To measure the performance of the segmentation and classification techniques of DR the most general parameters like sensitivity, specificity, and accuracy are considered. Se \& Sp measurements are the proportion of all around the grouped vessel and non-vessel pixels, 
individually. Acc is the general measure giving the proportion of all absolute vessel pixels. These measurements are utilized to look at the nature of the outcomes got from the blood vessels, lesions segmentation, and classification.

$$
\begin{aligned}
& S e=\frac{T P}{T P+F N} \\
& S p=\frac{T N}{T N+F P} \\
& A c c=\frac{(T P+T N)}{(T P+F N+T N+F P)}
\end{aligned}
$$

\begin{tabular}{|c|c|c|c|c|c|}
\hline \# & $\begin{array}{l}\text { Segmentation } \\
\text { Techniques }\end{array}$ & Methods & Sn & $\mathbf{S p}$ & Acc \\
\hline 1. & $\begin{array}{l}\text { Lei Zhanga et al., } \\
\qquad 2015\end{array}$ & $\begin{array}{c}\text { Multi-scale Gabor filter, k- } \\
\text { means }\end{array}$ & 0.7812 & 09668 & 0.9504 \\
\hline 2. & $\begin{array}{l}\text { R. GeethaRamani } \\
\text { et al., } 2016\end{array}$ & $\begin{array}{l}\text { Principal Component } \\
\text { Analysis and k-means }\end{array}$ & 0.7079 & 0.9778 & 0.9536 \\
\hline 3. & $\begin{array}{l}\text { Yu Qian Zhao et } \\
\text { al., } 2014\end{array}$ & Level set and region growing. & 73.54 & 97.89 & 94.77 \\
\hline 4. & $\begin{array}{c}\text { Argyrios } \\
\text { Christodoulidis et } \\
\text { al., } 2016\end{array}$ & $\begin{array}{l}\text { Line detection and perceptual } \\
\text { organization }\end{array}$ & 0.8506 & 0.9582 & 0.9479 \\
\hline 5. & $\begin{array}{l}\text { Sudeshna Sil Kar } \\
\text { et al., } 2016\end{array}$ & Fuzzy conditional entropy & 0.7632 & 0.9801 & 0.9628 \\
\hline 6. & $\begin{array}{c}\text { Shahab Aslani et } \\
\text { al., } 2016\end{array}$ & $\begin{array}{l}\text { B-COSFIRE filter, Random } \\
\text { forest classifier }\end{array}$ & 0.7545 & 0.9801 & 0.9648 \\
\hline 7. & $\begin{array}{l}\text { Nagendra Pratap } \\
\text { Singh et al., } 2016\end{array}$ & $\begin{array}{c}\text { Principal Component } \\
\text { Analysis }\end{array}$ & 0.7594 & 0.9801 & 0.9522 \\
\hline 8 & $\begin{array}{c}\text { Shailesh Kumar et } \\
\text { al., } 2020\end{array}$ & $\begin{array}{l}\text { Radial basis function neural } \\
\text { network }\end{array}$ & 0.8700 & 0.9300 & - \\
\hline \multicolumn{2}{|c|}{ Proposed Technique } & $\begin{array}{c}\text { AHE, Hop Field Neural } \\
\text { Network, ART-1 }\end{array}$ & 0.9998 & 0.9997 & 0.9999 \\
\hline
\end{tabular}

Table 2 Proposed segmentation results vs. existing segmentation techniques. 
As per Table 2, the proposed algorithm outperforms other deep learning techniques. The inherent consideration is the recurrent neurons are trained to adjust the image features to predict a small object that necessitates more contexts. The presentation of the proposed approach accomplished a normal estimation of $99.98 \%$ sensitivity, $99.97 \%$ specificity, and 99.99\% accuracy compared with competitive methods.

\section{CONCLUSION}

The detection of anomalies presented in the human eye's retina helps the physicians to conduct the dynamic procedure to diagnose DR. Utilization of the proposed indicator that diminishes the false negatives and gives dependable recognition precision in both position and mass size. The proposed system accurately detects both minor and major blood vessels and lesions from infected fundus images. Also, it detects the blood vessels that are even hard to diagnose cases, where many small and vague drusens are present in the retina. The proposed Hop Field Neural Network algorithm is an unsupervised method, reduces the probability of error occurred compared with other supervised methods. This algorithm segments the depigmented image and increases the exhibition of the system. The Adaptive Resonance Theory is included in the system is to classify the retinal blood vessel image and lesions to grade the level of diabetic retinopathy images obtained from the public databases and clinical images.

\section{ACKNOWLEDGMENTS}

The authors are thankful to the following medical experts: Dr. J. Kishore Kumar Jacob, Eye Specialist, K.K. Medical College Hospital, Nagercoil, India \& Dr. H. Hector, Consultant Ophthalmologist, C.S.I. Hospital, Neyyoor, India for providing all the required help to assess the segmentation results on clinical as well as public dataset fundus images. This research is done at the Center for Artificial Intelligence with the financial support by the Deanship of Scientific Research at King Khalid University under research grant number (RGP.1/210/42). 


\section{REFERENCES}

Varun Gulshan, Lily Peng, Marc Coram, Martin C Stumpe, Derek Wu, Arunachalam Subhashini, Kasumi, Tom Madams, Jorge, Ramasamy, Rajiv, Philip, Jessica, \& Dale R Webster. 2016. Development and Validation of a Deep Learning Algorithm for Detection of Diabetic Retinopathy in Retinal Fundus Photographs. JAMA. 29.

Francis D., \& T. Jemima Jebaseeli. 2016. Fundus image vessel segmentation using PCNN model. Proceedings of 2016 Online International Conference on Green Engineering and Technologies.

T. Jemima Jebaseeli, C. Anand Deva Durai, \& J. Dinesh Peter. 2018. Segmentation of Type - II Diabetic Patient's Retinal Blood Vessel to Diagnose Diabetic Retinopathy. Lecture Notes in Computational Vision and Biomechanics. 31: 268-286.

Gwenole Quellec, Mathieu Lamard, Pierre Marie Josselin, Guy Cazuguel, Beatrice Cochener, \& Christian RouxGwenole Quellec. 2008. Optimal Wavelet Transform for the Detection of Microaneurysms in Retina Photographs. IEEE Transactions on Medical Imaging. 27(9).

George Azzopardi, Nicola Strisciuglio, Mario Vento, \& Nicolai Petkov. 2015. Trainable COSFIRE filters for vessel delineation with application to retinal images. Medical Image Analysis, 19: 46-57.

Wilfred Franklin \& S. Edward Rajan. 2014. Computerized screening of diabetic retinopathy employing blood vessel segmentation in retinal images. Biocybernetics and biomedical engineering. 34: 117-124.

R. GeethaRamani \& Lakshmi Balasubramanian. 2016. Retinal blood vessel segmentation employing image processing and data mining techniques for computerized retinal image analysis. Biocybernetics and Biomedical Engineering. 36 (1): 102-118. 
Elaheh Imani, Javidi, \& Pourrez. 2015. Improvement of retinal blood vessel detection using morphological component analysis. Computer methods and programs in biomedicine. 118: 263-279.

E.Rajaby, S. M. Ahadi, \& H. Aghaeinia. 2016. Robust color image segmentation using fuzzy c-means with weighted hue and intensity. Digital Signal Processing. 51: 170-183.

Carla Agurto, Honggang Yu, Victor Murray, Marios S. Pattichis, Sheila Nemeth, Simon Barriga, \& Peter Soliz. 2015. A multiscale decomposition approach to detect abnormal vasculature in the optic disc. Computerized Medical Imaging and Graphics. 43:137-149.

Shailesh Kumar, AbhinavAdarsh, BasantKumar, \& Amit Kumar Singh. 2020. An automated early diabetic retinopathy detection through improved blood vessel and optic disc segmentation. Optics \& Laser Technology. 121.

Nagendra Pratap Singh \& Rajeev Srivastava. 2016. Retinal blood vessels segmentation by using Gumbel probability distribution function based matched filter. Computer methods and programs in biomedicine, 129: 40-50.

Shahab Aslani \& Haldun Sarnel. 2016. A new supervised retinal vessel segmentation method based on robust hybrid features. Biomedical Signal Processing and Control. 30:1-12.

Sudeshna Sil Kar \& Santi P. Maity. 2016. Retinal blood vessel extraction using tunable bandpass filter and fuzzy conditional entropy. Computer methods and programs in biomedicine, 133:111-132.

Argyrios Christodoulidi, Thomas Hurtut, Houssem Ben Tahar, \& Farida Cheriet. 2016. A multi-scale tensor voting approach for small retinal vessel segmentation in high resolution Fundus images. Computerized Medical Imaging and Graphics. 52:28-43. 
Yu Qian Zhao, Xiao Hong Wang, Xiao Fang Wang, \& Frank Y.Shih. 2014. Retinal vessels segmentation based on level set and region growing. Pattern Recognition. 47: 24372446.

Lei Zhanga, Mark Fisher, \& Wenjia Wang. 2015. Retinal vessel segmentation using multiscale textons derived from key points. Computerized Medical Imaging and Graphics. $45: 47-56$.

Zhaobin Wang, Shuai Wang, Ying Zhu, Yide Ma. 2016. Review of Image Fusion Based on Pulse-Coupled Neural Network. Archives of Computational Methods in Engineering. 23: 659-671. 